\title{
CONE-8309144--3
}

\section{OPERATOR INTERFACE TO THE ORIC CONTROL SYSTEM}

\author{
C. A. Ludemann and B. J. Casstevens \\ Oak Ridge National Laboratory* \\ Oak Ridge, Tennessee, USA
}

CONE-8309144--3

DEQ4 4000335

\section{Introduction}

The Oak Ridge Isochronous Cyclotron (ORIC) was built in the early 1960s with a hard-wired manual control system. Presently, it serves as a variable-energy heavyion cyclotron with an internal ion source, or as an energy booster for the new $25 \mathrm{MV}$ tandem electrostatic accelerator of the Holifield Heavy Ion Facility. One factor which has kept the cyclotron the productive research tool it is today is the gradual transfer of its control functions to a computer-based system beginning in the 1970 s.

This particular placement of a computer between an accelerator and its operators afforded some inique challenges and opportunities that would not be encountered today. Historically, the transformation began at a time when computers were just beginning to gain acceptance as reliable operational tools. Veteran operators with tens of years of accelerator experience justifiably expressed skepticism that this "improvement" would aid them, particularly if they had to re-learn how to operate the machine. The confidence of the operators was gained when they realized that one of the primary principles of ergonomics was being upheld. The computer software and hardware was being designed to serve them and not the computer.

The undertaking, in fact, was aided by information not usually available when one designs a new accelerator and control system simultaneously. The idiosyncracies of the accelerator were well-known and the areas in which the computer could provide assistance were readily identifiable. Furthermore, the operators had developed "natural" tuning techniques that a system designer would not necessarily have thought of. Typically, in a new installation these aspects are discovered only after a few years of operating experience. It is usually within the first revision of the software (but hopefully not the hardware) that trouble spots are eliminated and unanticipated features added.

The ORIC computer control system manages the operation of a moderate number of elements ( 200). They were transferred to the control of the computer system gradually while working within the constraints of an active research program. In fact,

*Research sponsored by the Basic Energy Sciences Division, U.S. Department of Energy under contract W-7405-eng-26 with the Union Carbide Corporation. 
there are many elements that remain hard-wired today because it would not be cost effective to transfer them to the system uniess they are modified or expanded upun.

\section{The Operator Console}

The cyclotron operators communicate with the computer by means of an alphanumeric keyboard and a "manual" control panel. CRT displays and a voice synthesizer provide status information from the computer. While this approach to operatormachine interface is relatively standard, the details of the control panel are not.

Physical Layout. The elements of the operator interface were installed in one $100 \mathrm{~cm}$ "wing" of the existing U-shaped hard-wired console (Fig. 1). The components replaced approximately 40 potentiometers, 40 panel meters, and 80 switches. The hard-wired console had been designed to minimize operator fatigue by making all controls readily visible and accessibie. The computer components were organized to conform to this example. Not shown in the foreground of the figure is a 30-cm horizontal shelf that permits the operator to rest log books or armis while tuning the machine. This shelf is $77 \mathrm{~cm}$ above the floor with adequate clear space for legs and feet. The :iJ cm by $48 \mathrm{~cm}$ panels that hold the alphanumeric keyboard and one portion of the control panel are inclined approximately 10 degrees above horizontal. The CRT faces and current metering panel are almost vertical. The $11 \mathrm{~cm}$ transition piece that supports hard-wired controls above the keyboard on the left, and the second portion of the control panel on the right, are inclined approximately 35 degrees.

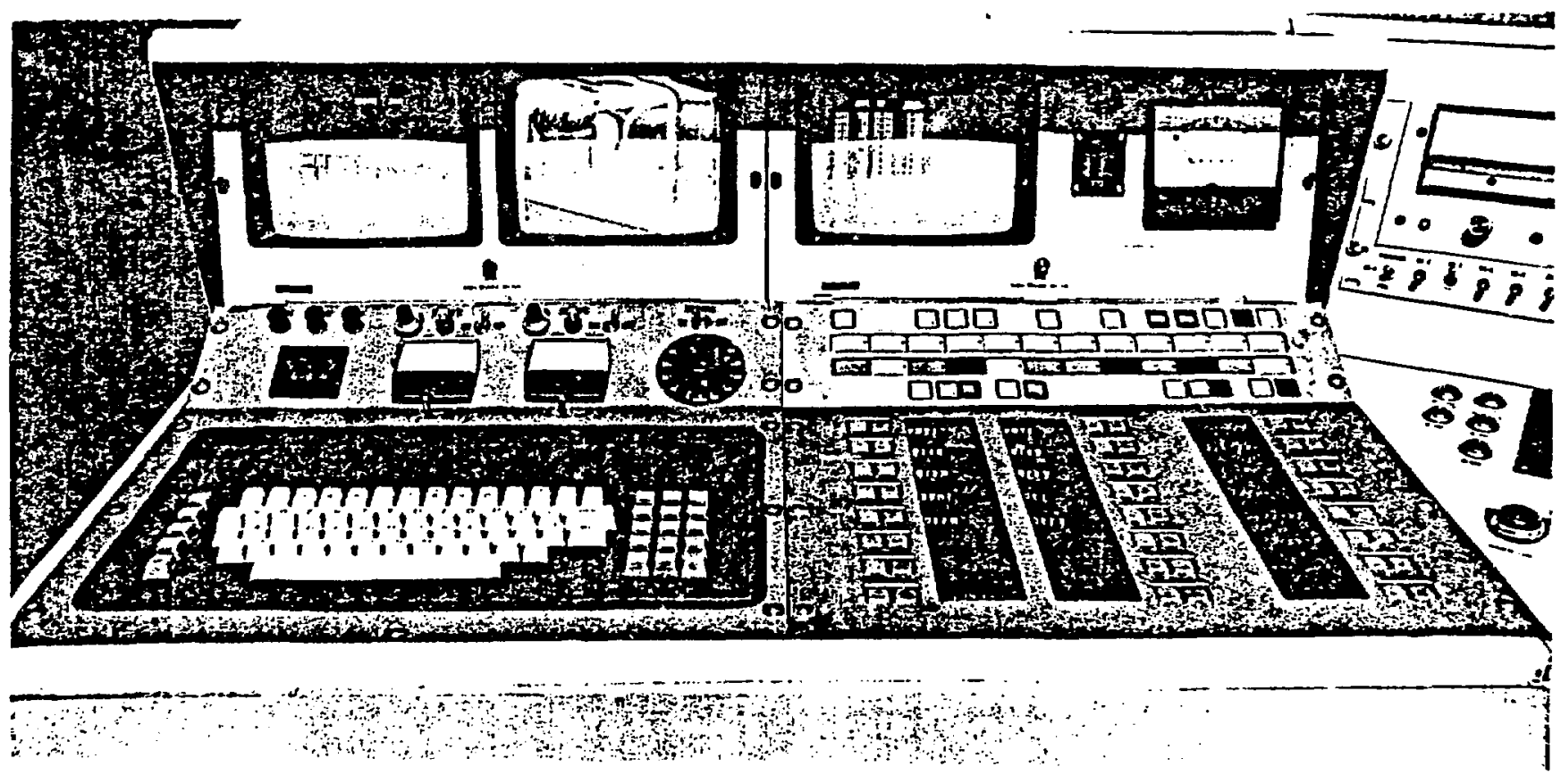

Figure 1. The components of the ORIC operator's computer interface mounted in the existing control console. 
Since the console height is only $112 \mathrm{~cm}$ above the floor, the operator has an almost unobstructed view of the remainder of the control room. The shield that was placed above the CRTs to reduce their illumination by oyerhead room lighting, also serves as a convenient shelf for resting materials during conversations between the operators and other staff members.

CRTs. The three $23 \mathrm{~cm}$ (diagonal measure) monochromatic CRTs are refreshed by CAMAC modules. Each screen is capable of presenting 241 ines of 64 alphanumeric characters. This information is easily readable by most operators at the console. Some difficulty in reading has been experienced, however, by a staff member who uses trifocal spectacles. Intensity variation and blinting characters are used rather than color to inform operators of equipment status and error conditions. This is because the fringe magnetic fieid of the cyclotron is of the order of $1-2 \mathrm{mTes} l a$ in this area of the control room. The left-hand CRT records major equipment malfunctions detected by the system, echoes characters from the keyboard, and displays "prompt questions" and reply messages from programs that the operator has entered by means of the keyboard. The center CRT displays equipment status or can be switched to monitor television images of equipment in the experimental caves. The third CRT presents the status of equipment that the operator is tuning with the control panel.

Control Panel. In order to tune the accelerator, operators need to have a large number of controls available to them rapidly and in logical order. Menu-picking and cursor manipulation to assign controls were considered to be undesirable distractions to the turing process and were to be avoided. These considerations led to the design of a panel comprised entirely of L-O-lighted and LED-labelea pushbutton switches.

The upper portion of the control panel has four rows of LED-lighted switches. On the tcp row are the "command" switches, the next row is for "page selection", the third row performs functions that modify the format of the CRT pages or the control page that has been selected, and the final row determines the rate and size of changes that will be performed by the controls on the lower portion of the panel. This last row also furnishes "save" and "restore" capabilities.

Eleven of the twelve page selection switches permit the operator to rapidiy select functional groups of controls. The left to right order of these switches corresponds to sub-systems that accelerate or transport the ion bean from the center of the ORIC to the final target station. The controls selected by two of the switches are determined by target station information entered by the operator via the keyboard. The beam from the ORIC can be directed to 14 different areas, and the tandem beam to 8 stations under control of this system.

The depression of a page switch selects up to 16 operating parameters (coil currents, coefficients of functional relationships, etc.) for operator manipulation. The name of each control (up to eight characters) appears in LEDs next to a pair of pushbutton switches in the two leftmost columns of switches on the lower portion of the panel. Each pair of switches becomes the "manual" control for a parameter. 
These controls appear on the panel in the order of beam transmission, alternating from left to right columns. The switches are recessed below the surface of the panel so they will not be depressed inadvertently by placement of books or arms on the console. The LED-labels are not coplanar with the red plastic covers but are mounted at an angle to permit easier viewing by the operator seated at the console.

The labeled switches permit the operator to raise or lower the currents in magnets, move motor-driven elements in or out, etc. The sense, or direction, of control is that the left (red) switch generally increases the value of the operating parameter, and the right (blue) switch decreases it. The rate and increment of change is determined by the states of the LED-lighted switches on the fourth row of the upper portion of the control panel. Changes occur once or sixteen times per second and can have three magnitudes: one bit, approximately one quarter percent full scale, and one percent full scale. The actual magnitude of change depends on the element being controlled. The rate and increment size switches make it possible for the operator to change the current from a power supply from zero to full output in as little as six seconds, or as long as 4.3 minutes if the supply is driven by a 12-bit DAC.

The settings of all parameters on a control page can be "saved" by depressing a LED-lighted switch next to the increment rate and size switches. The settings for each page are saved independently and remembered as the operator moves from page to page. If a parameter is altered from its saved setting the difference is recorded on the CRT next to its present setting. An operator may return a single element to its saved value by adjusting its controls to make the difference zero, or may restore al 1 elements on a page to their saved values by depressing the switch next to the save switch - the "restore" switch.

The column of eight pairs of LED labeled switches on the right side of the control panel allows the operator to control any 8 functions regardless of the page that is selected on the left portion of the control panel. These controls are assigned by use of the keyboard and have an independent set of rate, increment size, save, and restore switches.

The top row of LED-lighted switches represents commands that can be performed on most of the other switches on the panel. Once depressed these commands remain active for up to three seconds as indicated by their flashing LEDs. For example, by touching the "POF" command key and then, within three seconds, a page selection key, all power supplies associated with that page are slewed to zero current and turned off. Similarly, the operator can touch POF and a single switch on the control panel and that individual supply will be turned off. Other command keys that affect power supplies include:

ZAP - set the reference voltage to zero and turn the supply off immediateiy,

PON - turn the power supply on,

RSET - slew the supply's current to its value when last POFed or ZAPed. Other command keys are used to open and close vacuum valves, etc. 
The twelfth page selection key is labeled "ALL". It is used in conjunction with the command keys to affect all elements connected to the system. As an example, depressing ZAP and then ALL effectively SCRAMs the accelerator.

Keyboard. The alphanumeric keyboard is used to communicate with tasks that are entered by the use of keywords. These tasks permit the operator to ramp any power supply to a prescribed current, calculate the beam energy, file all the operating parameters on disk storage, search for and set the machine up from recorded settings, etc. All information entered through the keyboard is examined for commands (i.e., ZAP, POF, etc.) before it is passed to the task. This preprocessing permits the operator to perform critical operations (e.g., ZAP ALL) at the keyboard regardless of the task being executed. To limit the keystrokes needed to perform these command sequences, the function keys on the left side of the unit have the same labels as the command switches on the control panel and the keypad on the right side includes the names of all the page selection switches.

\section{Use of the Control Panel}

The design goal of any control system, hard-wired or computer-assisted, is to have the resulting system support the operating process and not confuse it. In describing the operator's console in the previous section it would appear that the principle just mentioned was being violated. The transformation of single-turn, ten-turn, and three-decade potentiometers into pushbutton switches was the initial concern of the operators and system designers as well. The concept was proposed because it provided the high density of well labeled controls the operators desired. Furthermore, it would eliminate the awkward and tiring arm and wrist positions necessary to tune with an individual potentiometer or shaft encoder without inadvertently disturbing others. In order to test the approach, a single trimming coil power supply was operated using a panel with a pair of control switches. When it was found that the operators had no difficulty adjusting the supply, a panel with rate, increment size, and twelve pairs of control switches was constructed. The ten trimming coil power supplies were controlled with this panel. The question as to whether the new panel led to confusion was in reality the question whether the machine could be tuned as well, or better, than with the hard-wired controls. The answer was "as well". The operators wished to know why so much effort was being expended to do something they could do previously. Their question was answered when the panel was used to control the harmonic coil power supplies.

The ORIC has three sets of three pairs of windings that are used to cancel imperfections in the magnetic field caused by asymmetric cyclotron elements such as the extraction system. Proper tuning of each set requires adjustment of their currents in such a manner that their contribution to the azimuthally averaged field is a constant while their resultant cancellation field can be altered in strength and azimuth. This was accomplished in the hard-wired system by modifying the voltage references to the electrically floating power supplies. Batteries, linear poten- 
tiometers, and fotentiometers whose resistance was proportional to the SINE of the angle of their shaft rotation were used. Reproducible operation was possible providing the coupling between potentiometer shafts remained fixed and the batteries were new. Under the computer system, the same strength, azimuth, and average field level controls were provided. However, the data values sent to the DACs providing the reference voltages to the supplies were generated by the appropriate trigonometric expressions modeling the process. The results were a significant increase in reliability, reproducibility, and resolution in control of the harmonic coils. It was at this point that the final version of the control panel could be completer and installed in the control console. It was the computer's computational capability that provided the endorsement from the operators.

It is interesting to observe the operators fine tune the ORIC today. Their concentration is directed primarily to the beam current metering panel and the video display of diagnostic devices, as it should be. They can move rapidly from one control.subsystem to another as they work the beam through the machine to the experimental station. It often appears that a Braille system is being used by the operator as his hands move down the lower panel balancing one control element against another. The fear of "getting lost" has almost disappeared because of the save and restore features. This lack of intimidation is unfortunate in some instances. If these features are used indiscriminately, one can rapidly detune the machine from a precalculated configuration with little hope of return.

\section{Functional Control - Additional Remarks}

Since the original implementation of the harmonic coil controls, other elements of the ORIC have been linked by functional relationships. There are two comments to be made which might be helpful to others who are about to attempt such a control strategy. First, the use of pushbutton switches to increment and decrement functional parameters provides a software advantage over an absolute control (e.g., absolute shaft encoder). If the operator attempts to exceed the range of a parameter, further requests for change can be ignored easily. With absolute devices it is necessary to maintain the new "dial readirig" in RAM or in the external hardware. Second, regardless of the hardware method, when functional parameters are used as controls, the designer should always provide means for the operator to break the relationship. As with the harmonic control outlined above, if a power supply fails, the operator must be able to control that supply for testing and repair purposes. In our system, the supply can be assigned to one of the right hand pairs of control switches. When this is done provision must be made in the software to calculate the new functional parameters using inverse relationships (even if they are outside what would be considered normal bounds). Another equally practical reason for permitting the relaxation of furictional control, especially during its original implementation, is to provide the accelerator physicists the ability to test the validity of the function itself. 


\section{Concluding Remarks}

The considerations that went into the design of the human interface for one of the first applications of a computer to the control of a cyclotron have been described.' Space does not permit an outline of the error detection and reporting schemes, the file management approach taken for the recording of operating data and automatic setup of the machinel), or the extensive software for the operator interaction with tasks entered by means of the keyboard.

Perhaps the most important practice exercised in this undertaking is one that is valid for the design of any new system: It is imperative that the system designer work in close communication with the operating staff through all stages of the project. After all, they are the ones that must use the system day after day. The success of the entire operation rests upon their ability to operate the machine.

\section{Acknowledgements}

The authors wish to express their appreciation for the early contributions by S. W. Mosko and J. M. Domaschko of the Physics Division, and E. Madden and the late E. McDaniel of the Instrumentation and Controls Division to this endeavor. We wish to thank also C. L. Viar and the entire ORIC operations staff, past and present, for their patience and constructive criticism and suggestions.

\section{Reference}

1. B. J. Casstevens and C. A. Ludemann, Database Automation of Accelerator Operation, ORNL/CSD/TM-191. 